\title{
Results of intraoperative radiotherapy given as a boost after breast conserving-surgery
}

\author{
Kraiwut Onthong ${ }^{1}$, Chakapong Chakkabat ${ }^{1,2}$, Chonnipa Nantavithya ${ }^{1,2}$, Kanjana Shotelersuk ${ }^{2}$, \\ Sikrit Denariyakoon ${ }^{3}$, Mawin Vongsaisuwon ${ }^{4}$, Adhisabandh Chulakadabbav ${ }^{3}$, Kris Chatamra ${ }^{3}$, \\ Kitwadee Saksornchai ${ }^{1,2}$
}

${ }^{1}$ Division of Therapeutic Radiology and Oncology, Department of Radiology, King Chulalongkorn Memorial Hospital, Thai Red Cross Society, Bangkok, Thailand; ${ }^{2}$ Division of Therapeutic Radiology and Oncology, Faculty of Medicine, Chulalongkorn University, Bangkok, Thailand; ${ }^{3}$ The Queen Sirikit Center for Breast Cancer, King Chulalongkorn Memorial Hospital, Thai Red Cross Society, Bangkok, Thailand; ${ }^{4}$ Department of Surgery, Faculty of Medicine, Chulalongkorn University, Bangkok, Thailand

Contributions: (I) Conception and design: K Onthong, K Saksornchai; (II) Administrative support: K Saksornchai; (III) Provision of study materials or patients: K Onthong; (IV) Collection and assembly of data: K Onthong, K Saksornchai; (V) Data analysis and interpretation: K Onthong, K Saksornchai; (VI) Manuscript writing: All authors; (VII) Final approval of manuscript: All authors.

Correspondence to: Kitwadee Saksornchai. Division of Therapeutic Radiology and Oncology, Faculty of Medicine, Chulalongkorn University, Bangkok 10330, Thailand. Email: Kitwadee.s@chula.ac.th.

Background: Whole breast irradiation after breast-conserving surgery (BCS) with an external beam boost of 10-16 Gy is currently the standard treatment in breast cancer. Various modalities have been used for tumor bed boost irradiation. This study aimed to evaluate the local recurrence rate, overall survival rate (OSR), toxicity and cosmetic outcome of intraoperative radiotherapy (IORT) as a boost followed by whole breast irradiation in patients who received BCS.

Methods: This is a retrospective study. Between December 2009 and March 2017, 81 patients who underwent BCS with IORT as a boost were enrolled in this study. For IORT, a single dose of 20 Gy was delivered using a 30-50 kV photon beam, intraoperatively. All patients received whole breast radiation therapy (WBRT) of 42.5-50 Gy over 4-5 weeks. The primary endpoint was a 3-year local recurrence rate. Secondary endpoints included the OSR, toxicity and cosmetic outcome at 6 months after radiation treatment.

Results: At a median follow-up of 43 months, ipsilateral local recurrence was observed in one of the 81 patients $(1.2 \%)$ which occurred in the same quadrant of the breast index. The 3 -year OSR was $89.8 \%$. Treatment was well-tolerated with no grade 3-4 acute and late toxicity, and $87 \%$ of patients were recorded as excellent-good cosmesis.

Conclusions: The use of BCS with IORT as a boost resulted in a low local recurrence rate and excellent cosmetic outcome in early breast cancer. Thus, IORT as a boost could be considered as an alternative to an external beam boost. Prospective studies are needed to confirm this data.

Keywords: Intraoperative radiotherapy (IORT); boost; early breast cancer; local recurrence; cosmetic outcomes; whole breast irradiation

Submitted Feb 22, 2020. Accepted for publication Aug 06, 2020.

doi: 10.21037/gs-20-249

View this article at: http://dx.doi.org/10.21037/gs-20-249 


\section{Introduction}

Radiotherapy after breast-conserving surgery (BCS) has shown to be equivalent, in terms of survival outcomes to mastectomy (1-9). The traditional radiation involves 5 weeks of whole breast irradiation with 5-8 fractions of tumor bed boost. According to two studies, the Lyon and EORTC 22881-10882 trials $(10,11)$ tumor bed boost after BCS was effective in reducing the risk of ipsilateral breast recurrence. Tumor bed boost irradiation can be achieved by various techniques such as interstitial brachytherapy, external beam radiotherapy and intraoperative radiotherapy (IORT). IORT could be performed with either electron beams or photon beams and delivers a single dose radiation therapy to the tumor bed during surgery. A few studies have shown that, in terms of the local recurrence, overall survival rate (OSR) and wound complication, IORT given as a boost after BCS had much the same result as whole breast radiation therapy (WBRT) followed by an external beam radiation boost (12-14).

We assessed the local recurrence, OSR, complication and cosmetic outcome in early breast cancer patients who received the low energy photon intraoperative boost irradiation after BCS in our institution. We present the following article in accordance with the STROBE reporting checklist (available at http://dx.doi.org/10.21037/gs-20-249).

\section{Methods}

\section{Study design}

We conducted a retrospective study from December 2009 to March 2017 involving 138 patients with early breast cancer who underwent BCS with IORT as a boost in King Chulalongkorn Memorial Hospital (KCMH). Eligibility criteria consisted of early breast cancer (T1-T2, N0-N1), unifocal lesion and a negative pathological margin. Patients with an Eastern Cooperative Oncology Group (ECOG) higher than 2, who underwent re-excision or mastectomy after IORT from positive margin or with gross residual disease, or who had a history of previous thoracic/breast irradiation were excluded.

\section{Radiation treatment planning and technique}

In all patients, wide local excision of the tumor was performed with a clear resected margin. Sentinel lymph node biopsy or axillary lymph node dissection was performed during the procedure. The low energy photon
(INTRABEAM 600, Carl Zeiss Meditec AG, Oberkochen, Germany) was used in the surgical cavity immediately following tumor removal. A single 20-Gy dose was applied at the surface applicator and attenuated to 5-7 Gy at $1 \mathrm{~cm}$ depth, using a $30-50 \mathrm{KV}$ photon beam over $30 \mathrm{~min}$. All patients received post-operative external beam radiation (EBRT) to the conserved breast tissue and/or regional lymph nodes at a dose of 42.5-50 Gy using a conventional or three dimensional planning technique without an additional tumor bed boost. For WBRT, all cases were treated with an opposing tangential field in a supine position, arm above the head using $6 \mathrm{MV}$ photon.

\section{Assessment of endpoint}

The primary endpoint of this study was the 3-year local recurrence rate. Secondary endpoints were the 3-year OSR, acute/late toxicities and cosmetic outcomes. Acute and late toxicities were evaluated according to Common Terminology Criteria for Adverse Events, Version 3.0 (CTCAE). Acute toxicity was evaluated at the end of WBRT and late toxicity was evaluated at 1 and 3 years after WBRT. Cosmetic outcome was evaluated at least 12 months after radiation using the Harvard/NSABP/RTOG Breast Cosmesis Grading Scale. Photographs of patients were taken in two positions, two arms up above head and arm at the waist. All photographs were assessed by the panel of three radiation oncologists and two surgeons. The OSR was defined as the length of time after BCS until death from any cause. Local recurrence rate was defined as recurrence in ipsilateral breast and proved by surgical pathology.

Statistical analysis was performed with SPSS 22.0 statistical software. The OSR and local recurrence rate were estimated using the Kaplan-Meier method. Frequency analysis was used for acute toxicity, late toxicity, and cosmetic outcome.

The study was conducted in accordance with the Declaration of Helsinki (as revised in 2013). The study protocol was approved by the Institutional Review Board of King Chulalongkorn Memorial Hospital (Number 060/60) and individual consent for this retrospective analysis was waived.

\section{Results}

Between December 2009 and March 2017, 138 patients with early breast cancer who received IORT in KCMH were initially enrolled in this study. A total of 57 patients 
Table 1 Patients and tumor characteristics

\begin{tabular}{|c|c|}
\hline Characteristic & $\mathrm{N}(\%)$ \\
\hline \multicolumn{2}{|l|}{ Age } \\
\hline $30-40$ & $6(7.4)$ \\
\hline $41-50$ & $24(29.6)$ \\
\hline $51-60$ & $30(37.0)$ \\
\hline $61-70$ & $14(17.3)$ \\
\hline$>70$ & $7(8.7)$ \\
\hline \multicolumn{2}{|l|}{ Side } \\
\hline Right & $42(51.9)$ \\
\hline Left & $39(48.1)$ \\
\hline \multicolumn{2}{|l|}{ Tumor size $(\mathrm{cm})$} \\
\hline $0-1.0$ & $12(14.8)$ \\
\hline $1.1-2.0$ & $32(39.5)$ \\
\hline $2.1-3.0$ & $31(38.3)$ \\
\hline $3.1-4.0$ & $5(6.2)$ \\
\hline $4.1-5.0$ & $1(1.2)$ \\
\hline \multicolumn{2}{|l|}{ Nodal status } \\
\hline No & $63(77.8)$ \\
\hline $\mathrm{N} 1$ & $16(19.8)$ \\
\hline $\mathrm{Nx}$ & $2(2.4)$ \\
\hline \multicolumn{2}{|l|}{ Histology } \\
\hline Invasive ductal carcinoma & $76(93.8)$ \\
\hline Mucinous carcinoma & $2(2.4)$ \\
\hline Invasive papillary & $2(2.4)$ \\
\hline Invasive mammary & $1(1.4)$ \\
\hline \multicolumn{2}{|l|}{ Tumor grade } \\
\hline Gr 1 & $15(18.5)$ \\
\hline Gr 2 & $33(40.8)$ \\
\hline
\end{tabular}

Table 1 (continued)

were excluded (16 patients received IORT alone, 15 received an additional electron boost after IORT, 11 underwent mastectomy or re-excision after IORT, seven were lost in follow-up, two were diagnosed with pure ductal carcinoma in situ (DCIS), one had a tumor size of more than $5 \mathrm{~cm}$ and one was at the $\mathrm{N} 2$ disease stage. Thus, after their exclusion, a total of 81 patients were included in the trial analysis. The median follow up time was
Table 1 (continued)

\begin{tabular}{|c|c|}
\hline Characteristic & N (\%) \\
\hline Gr 3 & $29(35.8)$ \\
\hline Unknown & $7(8.6)$ \\
\hline \multicolumn{2}{|c|}{ Margin status, $\mathrm{cm}$} \\
\hline$<0.1$ & $10(12.3)$ \\
\hline $0.1-0.2$ & $29(35.8)$ \\
\hline $0.3-0.5$ & $23(28.4)$ \\
\hline$>0.5$ & $12(14.8)$ \\
\hline Unknown & $7(8.6)$ \\
\hline \multicolumn{2}{|c|}{ Lymphovascular invasion } \\
\hline Positive & $20(24.7)$ \\
\hline Negative & $56(69.1)$ \\
\hline Unknown & $5(6.2)$ \\
\hline \multicolumn{2}{|l|}{ ER } \\
\hline Positive & $58(71.6)$ \\
\hline Negative & $23(28.4)$ \\
\hline \multicolumn{2}{|l|}{ PR } \\
\hline Positive & $54(66.7)$ \\
\hline Negative & $27(33.3)$ \\
\hline \multicolumn{2}{|l|}{ HER 2} \\
\hline Positive & $11(13.6)$ \\
\hline Negative & $70(86.4)$ \\
\hline \multicolumn{2}{|l|}{ Chemotherapy } \\
\hline Yes & $57(70.4)$ \\
\hline No & $24(29.6)$ \\
\hline \multicolumn{2}{|l|}{ Hormonal Tx } \\
\hline Yes & 55 (67.9) \\
\hline No & $26(32.1)$ \\
\hline
\end{tabular}

43 months (range, 7-87 months). Baseline patients and tumor characteristics are shown in Table 1.

The median patients' age was 54 years old (range, 35-81 years). The median size of the tumor was $1.9 \mathrm{~cm}$. In total, 16 patients $(19.8 \%)$ had metastatic axillary lymph nodes, while $15(18.5 \%), 33(40.8 \%)$ and $29(35.8 \%)$ patients had tumor grade 1, 2 and 3, respectively. In terms of WBRT, only one patient received hypofractionation 
Table 2 Acute and late skin toxicities of the patients in the study

\begin{tabular}{|c|c|c|c|c|c|c|c|c|c|}
\hline Group of patients & \multicolumn{3}{|c|}{ Acute toxicity, N (\%) } & \multicolumn{3}{|c|}{ Late toxicity at 1 year, $\mathrm{N}(\%)$} & \multicolumn{3}{|c|}{ Late toxicity at 3 years, N (\%) } \\
\hline Received chemotherapy for & & $\mathrm{N}=57$ & & & $\mathrm{~N}=57$ & & & $\mathrm{~N}=52$ & \\
\hline 3-6 months & 19 (33.3) & $32(56.2)$ & $6(10.5)$ & $24(42.1)$ & $33(57.9)$ & 0 & $8(15.4)$ & $37(71.1)$ & $7(13.5)$ \\
\hline No adjuvant chemotherapy & & $\mathrm{N}=24$ & & & $\mathrm{~N}=24$ & & & $\mathrm{~N}=23$ & \\
\hline
\end{tabular}

irradiation which was 42.5 Gy in 16 fractions. A total of 57 (70.4\%) patients received chemotherapy and 55 (69.7\%) received hormonal therapy. Anthracycline and/or taxane based chemotherapy was prescribed for patients who were indicated. For the IORT procedure, the median treatment time was $26.4 \mathrm{~min}$ (range, 13.0-52.3 $\mathrm{min}$ ) and the median applicator size was $3.5 \mathrm{~cm}$ (range, $2.5-5 \mathrm{~cm}$ ).

One of the 81 patients $(1.2 \%)$ had ipsilateral breast tumor recurrence in the tumor bed which was occurred as the same quadrant as the primary tumor. This patient was triple-negative for breast cancer and pathological findings showed high grade invasive ductal carcinoma with an extensive intraductal component. Time to ipsilateral breast cancer recurrence was 78 months. The 3 -year OSR was $89.8 \%$. The acute and late toxicity were grade $0-2$ in all 81 patients, without grade $3-4$ toxicity being detected, according to CTCAE v3.0. One patient who received hypofractionation whole breast irradiation was noted grade 0 in acute and late toxicity. For acute toxicity, there are $74 \%$ of patients who were evaluated at the end of the WBRT, while the rest of those were evaluated at 1-2 months after the WBRT. At 3 years after radiation, $92.6 \%(\mathrm{~N}=75)$ of the patients were available for late toxicity. Grade 2 late toxicities include marked hyperpigmentation and subcutaneous fibrosis.

A total of 57 out of 81 patients (70\%) received adjuvant chemotherapy, receiving the chemotherapy after IORT and before WBRT, while the other $30 \%$ received WBRT approximately 3-4 weeks after IORT. The median time between IORT and WBRT was 4 months. The time interval between IORT and WBRT showed no significant association with late toxicities $(\mathrm{P}=0.46)$. The acute and late skin toxicity results are shown in Table 2 .

The cosmetic outcome was evaluated according to the Harvard/NSABP/RTOG breast cosmesis grading scale, where 71 patients (87\%) experienced excellentgood cosmesis. The median time of cosmetic evaluation is
41 months after the radiation and $74.1 \%$ of the patients was evaluated at more than 2 years after radiation.

\section{Discussion}

At present, post-operative EBRT to the whole breast followed by tumor bed boost is the standard method of care for early breast cancer. An additional tumor bed boost of 10-16 Gy significantly reduces the risk of local recurrence rate $(10,11)$. Despite the boost radiation, the majority of local recurrences still occurred close to the previous primary tumor bed (15), which might reflect a geographical miss of the tumor bed causing this local failure. The use of IORT, a form of APBI, can shorten the treatment time and reduce the level of geographical misses. Another biological advantage of IORT which was proposed by few studies is that the radiation is given at the earliest stage before the tumor cells have a chance to proliferate $(16,17)$.

In our study, at a median follow-up of 43 months, ipsilateral breast tumor recurrence occurred in one of the 81 patients $(1.2 \%)$ after receiving IORT as a boost, which was quite low compared to the previous studies from EORTC 22881-10882 and Lyon trials. In Lyon trial, the median follow up time was 3.3 years and the crude rate of local recurrence in the boost arm was $1.9 \%$ which was similar to our results. The median follow up period was longer than our study at 5 years for the EORTC 22881-10882 trial and the local recurrence rate was $4.3 \%$. Our local recurrence rate was also consistent with the other IORT boost studies (18-22). Those results are shown in Table 3.

The 3 -year OSR in this study was $89.8 \%$, which was not markedly different to in those in the Lyon (5-year OSR was 92.9\%) and EORTC 22881-10882 (5-year OSR of 91\%) trials $(10,23)$. The study from Germany also reported 3-year OSR which was $91.3 \%$ (18). Recently, 15 years outcome of patients receiving IORT as a boost was reported from Pez et al. (19). This retrospective study revealed the 15 
Table 3 Five-year IBRT of IORT boost studies (low energy X-ray)

\begin{tabular}{lccc}
\hline Studies & No. of patients & Median F/U time (months) & IBRT \\
\hline Blank et al. (2010) (18) & 197 & 37 & $3 \%(3-y e a r)$ \\
Pez et al. (2020) (19) & 400 & $78 \%(5-y e a r)$ & $6.6 \%(10$ year) \\
& & 34 (15 year) & $10.1 \%$ (3-year) \\
Wenz et al. (2010) (20) & 154 & 39 & No local recurrence \\
Chang et al. (2014) (21) & 55 & 60.5 & $1.73 \%(5-y e a r)$ \\
Vaidya et al. (2011) (22) & 299 & 43 & $1.2 \%(4-y e a r)$ \\
Our study & 81 & & 39 \\
\hline
\end{tabular}

IBRT, ipsilateral breast tumor recurrence; IORT, intraoperative radiotherapy.

years of local recurrence and OSRs were $10.1 \%$ and $80.7 \%$, respectively.

In terms of toxicity, our study showed mild acute and late toxicity, without severe grade 3 or grade 4 toxicity and did not differ from others. Although some of the patients received chemotherapy after IORT, the toxicities were similar to those who did not receive chemotherapy.

Numerous studies have assessed the toxicity from a similar treatment. Late toxicity after 3 years was evaluated as mild in most of the patients and $6 \%$ of those had grade 3 toxicity from Wenz et al. (20). Similarly, Chang identified $2 \%$ of patients who had grade 3 late skin toxicity (21). In this study, they reported the worst score during every 6 month follow up time for 3 years which was different from our study that reported late toxicity at a specific point of time. Pez et al. reported fibrosis as the most common late toxicity which accounted for $10 \%$ at 1 year and increased to $19 \%$ at 5 years (19).

The cosmetic outcome in our study was rated as excellent-good, according to the Harvard/NSABP/ RTOG breast cosmesis grading scale. Kraus-Tiefenbacher et al. (24) reported an excellent-good cosmetic outcome at 4-6 months after surgery with IORT in about $90-95 \%$ of all patients. The EORCT 22881-10882 trial showed 71\% of the patients in the boost group had an excellent or good cosmetic outcome at 3 years (25) which was similar to our results.

The results of intraoperative electron boost (IOERT) followed by whole breast irradiation have also been published. The International Society of Intraoperative Radiotherapy (ISIORT) revealed the most long-term analysis at a median follow-up of 72 months. The inbreast tumor control rate was $99.2 \%$, which $50 \%$ of local recurrence tumor was occurred at the same quadrant of the initial one (26). A matched-pair study was performed by comparing patients receiving external beam boost with IOERT (15). The 5 -year local recurrence rate was $4.3 \%$ and $0 \%$ in the arm of external beam and IOERT, respectively. This study also showed $93 \%$ of patients experienced satisfactory and $98 \%$ with acceptable cosmetic outcome at 45 months follow up. Currently, an ongoing TARGIT IORT boost trial (27), a phase III randomized study is testing the superiority of IORT boost to the external beam boost. These results are awaited.

This study is a retrospective study, which resulted in limitations in data collection and may introduce a selection bias. The other limitation is the short follow-up time of 43 months and needed for long-term follow up for tumor control and cosmetic outcome assessment.

\section{Conclusions}

Lumpectomy with IORT as a boost followed by external beam radiation to the whole breast is feasible in terms of low local recurrence rate with minimal toxicity and good cosmetic outcome in early breast cancer. However, a prospective study and long term follow-up are required to validate this data.

\section{Acknowledgments}

Funding: None.

\section{Footnote}

Reporting Checklist: The authors have completed the 
STROBE reporting checklist. Available at http://dx.doi. org/10.21037/gs-20-249

Data Sharing Statement: Available at http://dx.doi. org/10.21037/gs-20-249

Peer Review File: Available at http://dx.doi.org/10.21037/gs20-249

Conflicts of Interest: All authors have completed the ICMJE uniform disclosure form (available at http://dx.doi. org/10.21037/gs-20-249). The authors have no conflicts of interest to declare.

Ethical Statement: The authors are accountable for all aspects of the work in ensuring that questions related to the accuracy or integrity of any part of the work are appropriately investigated and resolved. The study was conducted in accordance with the Declaration of Helsinki (as revised in 2013). The study protocol was approved by the Institutional Review Board of King Chulalongkorn Memorial Hospital (Number 060/60) and individual consent for this retrospective analysis was waived.

Open Access Statement: This is an Open Access article distributed in accordance with the Creative Commons Attribution-NonCommercial-NoDerivs 4.0 International License (CC BY-NC-ND 4.0), which permits the noncommercial replication and distribution of the article with the strict proviso that no changes or edits are made and the original work is properly cited (including links to both the formal publication through the relevant DOI and the license). See: https://creativecommons.org/licenses/by-nc-nd/4.0/.

\section{References}

1. Blichert-Toft M, Rose C, Andersen JA, et al. Danish randomized trial comparing breast conservation therapy with mastectomy: six years of life-table analysis. Danish Breast Cancer Cooperative Group. J Natl Cancer Inst Monogr 1992;11:19-25.

2. Fisher B, Anderson S, Bryant J, et al. Twenty year followup of a randomized trial comparing total mastectomy, lumpectomy, and lumpectomy plus irradiation for the treatment of invasive breast cancer. N Engl J Med 2002;347:1233-41.

3. Fisher B, Anderson S, Redmond CK, et al. Reanalysis and results after 12 years of follow-up in a randomized clinical trial comparing total mastectomy with lumpectomy with or without irradiation in the treatment of breast cancer. $\mathrm{N}$ Engl J Med 1995;333:1456-61.

4. Jacobson JA, Danforth DN, Cowan KH, et al. Ten year results of a comparison of conservation with mastectomy in the treatment of stage I and II breast cancer. $\mathrm{N}$ Engl J Med 1995;332:907-11.

5. Sarrazin D, Le MG, Arriagada R, et al. Ten-year results of a randomized trial comparing a conservative treatment to mastectomy in early breast cancer. Radiother Oncol 1989;14:177-84.

6. van Dongen JA, Voogd AC, Fentiman IS, et al. Long-term results of a randomized trial comparing breast-conserving therapy with mastectomy: European Organization for Research and Treatment of Cancer 10801 trial. J Natl Cancer Inst 2000;92:1143-50.

7. Veronesi U, Cascinelli N, Mariani L, et al. Twentyyear follow-up of a randomized study comparing breastconserving surgery with radical mastectomy for early breast cancer. N Engl J Med 2002;347:1227-32.

8. Veronesi U, Luini A, Galimberti V, et al. Conservation approaches for the management of stage I/II carcinoma of the breast: Milan Cancer Institute trials. World J Surg 1994;18:70-5.

9. Arriagada R, Le MG, Rochard F, et al. Conservative treatment versus mastectomy in early breast cancer: patterns of failure with 15 years of follow-up data. Institut Gustave-Roussy Breast Cancer Group. J Clin Oncol 1996;14:1558-64.

10. Romestaing P, Lehingue Y, Carrie C, et al. Role of a 10Gy boost in the conservative treatment of early breast cancer: results of a randomized clinical trial in Lyon, France. J Clin Oncol 1997;15:963-8.

11. Bartelink H, Maingon P, Poortmans P, et al. Whole-breast irradiation with or without a boost for patients treated with breast-conserving surgery for early breast cancer: 20year follow-up of a randomised phase 3 trial. Lancet Oncol 2015;16:47-56.

12. Njeh CF, Saunders MW, Langton CM. Accelerated Partial Breast Irradiation (APBI): A review of available techniques. Radiat Oncol 2010;5:90.

13. Reitsamer R, Peintinger F, Sedlmayer F, et al. Intraoperative radiotherapy given as a boost after breastconserving surgery in breast cancer patients. Eur J Cancer 2002;38:1607-10.

14. Lemanski C, Azria D, Thezenas S, et al. Intraoperative radiotherapy given as a boost for early breast cancer: long-term clinical and cosmetic results: long-term 
clinical and cosmetic results. Int J Radiat Oncol Biol Phys 2006;64:1410-5.

15. Reitsamer R, Peintinger F, Kopp M, et al. Local recurrence rates in breast cancer patients treated with intraoperative electron-boost radiotherapy versus postoperative externalbeam electron-boost irradiation. A sequential intervention study. Strahlenther Onkol 2004;180:38-44.

16. Herskind C, Steil V, Kraus-Tiefenbacher U, et al. Radiobiological aspects of intraoperative radiotherapy (IORT) with isotropic low-energy $\mathrm{X}$ rays for early-stage breast cancer. Radiat Res 2005;163:208-15.

17. Herskind C, Griebel J, Kraus TU, et al. Sphere of equivalence - a novel target volume concept for intraoperative radiotherapy using low-energy $\mathrm{X}$ rays. Int J Radiat Oncol Biol Phys 2008;72:1575-81.

18. Blank E, Kraus TU, Welzel G, et al. Single-center longterm follow-up after intraoperative radiotherapy as a boost during breast-conserving surgery using low-kilovoltage X-rays. Ann Surg Oncol 2010;17:352-8.

19. Pez M, Keller A, Welzel G, et al. Long-term outcome after intraoperative radiotherapy as a boost in breast cancer. Strahlenther Onkol 2020;196:349-55.

20. Wenz F, Welzel G, Blnak E, et al. Intraoperative radiotherapy as a boost during breast-conserving surgery using low-kilovoltage $\mathrm{X}$-rays: The first 5 years of experience with a novel approach. Int J Radiat Oncol Biol Phys 2010;77:1309-14.

21. Chang DW, te Marvelde L, Chua BH. Prospective study of local control and late radiation Toxicity after intraoperative radiation therapy boost for early breast cancer. Int J Radiat Oncol Biol Phys 2014;88:73-9.

22. Vaidya JS, Baum M, Tobias JS, et al. Long-term results of targeted intraoperative Radiotherapy (TARGIT) boost during breast-conserving surgery. Int J Radiat Oncol Biol Phys 2011;81:1091-7.

23. Bartelink H, Horiot JC, Poortmans P, et al. Recurrence rates after treatment of breast cancer with standard radiotherapy with or without additional radiation. $\mathrm{N}$ Engl J Med 2001;345:1378-87.

24. Kraus-Tiefenbacher U, Bauer L, Kehrer T, et al. Intraoperative Radiotherapy (IORT) as a Boost in Patients with Early-Stage Breast Cancer - Acute Toxicity. Onkologie 2006;29:77-82.

25. Vrieling C, Collette L, Fourquet A, et al. The influence of the boost in breast conserving therapy on cosmetic outcome in the EORTC "boost versus no boost" trial. Int J Radiat Oncol Biol Phys 1999;45:677-85.

26. Kaiser J, Reitsamer R, Kopp P, et al. Intraoperative electron radiotherapy (IOERT) in the treatment of primary breast cancer. Breast Care 2018;13:162-7.

27. ClinicalTrials.gov. A Comparison of Intra-operative Radiotherapy Boost With External Beam Radiotherapy Boost in Early Breast Cancer. (TARGIT-B) [cited 2020 June 2]. Available online: https://clinicaltrials.gov/ct2/ show/NCT01792726
Cite this article as: Onthong $\mathrm{K}$, Chakkabat C, Nantavithya C, Shotelersuk K, Denariyakoon S, Vongsaisuwon M, Chulakadabbav A, Chatamra K, Saksornchai K. Results of intraoperative radiotherapy given as a boost after breast conserving-surgery. Gland Surg 2020;9(5):1389-1395. doi: 10.21037/gs-20-249 